\title{
L’École de Quévillon dans la région de Lanaudière
}

\section{Serge Joyal}

Volume 54, 1987

Culture et religion dans la région de Lanaudière

URI : https://id.erudit.org/iderudit/1006966ar

DOI : https://doi.org/10.7202/1006966ar

Aller au sommaire du numéro

Éditeur(s)

Les Éditions Historia Ecclesiæ Catholicæ Canadensis Inc.

ISSN

0318-6172 (imprimé)

1927-7067 (numérique)

Découvrir la revue

Citer cet article

Joyal, S. (1987). L'École de Quévillon dans la région de Lanaudière. Sessions d'étude - Société canadienne d'histoire de l'Église catholique, 54, 117-129.

https://doi.org/10.7202/1006966ar

\section{Résumé de l'article}

Au cours de l'année 1975-1976, en compagnie du P. Wilfrid Corbeil, c.s.v., président fondateur du Musée d'art de Joliette, l'Hon. Serge Joyal visitait les églises, les presbytères et les couvents du diocèse de Joliette, en vue de mettre à jour l'inventaire des trésors des fabriques, dressé par Gérard Morissette et Germain Bazi, quelques cinquante ans auparavant. L'auteur livre, dans sa communication, les résultats de ces visites patientes et répétées qui lui ont permis de prendre connaissance des livres de compte et de délibérations des fabriques de la région de Lanaudière.
Tous droits réservés @ Les Éditions Historia Ecclesiæ Catholicæ Canadensis Inc., 1987
Ce document est protégé par la loi sur le droit d'auteur. L'utilisation des services d'Érudit (y compris la reproduction) est assujettie à sa politique d'utilisation que vous pouvez consulter en ligne.

https://apropos.erudit.org/fr/usagers/politique-dutilisation/ 


\title{
L'École de Quévillon dans la région de Lanaudière
}

\author{
L'Honorable Serge JOYAL, c.p.
}

\section{RÉSUMÉ}

Au cours de l'année 1975-1976, en compagnie du P. Wilfrid Corbeil, c.s.v., président fondateur du Musée d'art de Joliette, l'Hon. Serge Joyal visitait les églises, les presbytères et les couvents du diocèse de Joliette, en vue de mettre à jour l' inventaire des trésors des fabriques, dressé par Gérard Morissette et Germain Bazi, quelques cinquante ans auparavant. L'auteur livre, dans sa communication, les résultats de ces visites patientes et répétées qui lui ont permis de prendre connaissance des livres de compte et de délibérations des fabriques de la région de Lanaudière.

On pourrait aborder le sujet de multiples façons: retracer la biographie de Quévillon; faire la liste des églises et chapelles à l'ornementation desquelles il a été associé; identifier ses disciples, apprentis ou associés qui l'ont suivi dans la région; voir ce qui subsiste de l'œuvre sculpté; établir enfin les mesures à prendre pour protéger cet œuvre de la destruction et de l'oubli.

Louis-Amable Quévillon est né le 14 octobre 1749 et décédé, célibataire, le 11 mars 1823, à l'âge de 74 ans. On ne connaît aucun portrait du personnage. Précisons qu'il s'agit essentiellement dans cette communication de l'œuvre du sculpteur. Certes, Quévillon, né et élevé à St-Vincent de Paul, fut formé comme son frère Jean-Baptiste, maître menuisier, en suivant un apprentissage de 9 ans (comme il était courant à l'époque). On le décrit aussi comme entrepreneur; mais sa qualité essentielle, celle qui nous retient, est sa profession de sculpteur, le métier auquel il attachera son nom et des disciples en abondance.

\section{Le précurseur: Philippe Liébert (1733-1804)}

Le maître de Quévillon ne nous est pas connu avec certitude. Plusieurs indices donnent à penser qu'il s'agit de Philippe Liébert, sculpteur 
de Montréal, qui entreprit dans plusieurs églises un travail d'ornementation que compléta par la suite Quévillon. L'influence et les modèles stylistiques de Liébert sur Quévillon sont nettement évidents. De Liébert, l'on peut encore admirer la chaire qu'il sculpta pour l'église de L'Assomption en 1773 et le retable qu'il fit pour l'église de Repentigny en 1760-1761, heureusement sauvé de l'incendie de 1985, étant alors en restauration à l'extérieur. Liébert a également sculpté, entre 1791 et 1798, pour l'église de St-Cuthbert, un «autel à la romaine, une chaire et un banc d'œuvre». (Seul subsiste I'autel, conservé au Musée d'art de St-Laurent de Montréal).

\section{Le Bas-Canada à la fin du XVIII' ${ }^{e}$ siècle}

Pour bien comprendre le phénomène, il faut se situer dans le contexte de l'époque; c'est-à-dire à la fin du XVIII' siècle dans le Bas-Canada. Le régime britannique était bien installé; l'Acte de Québec de 1774 avait reconnu les droits et la liberté de culte aux Canadiens français; l'Acte constitutionnel de 1791 avait créé une chambre d'assemblée. Les Canadiens se multipliaient; le commerce du bois avec l'Angleterre se développait à la faveur des guerres napoléoniennes; des paroisses nouvelles étaient fondées, et, au fur et à mesure de leur expansion, on procédait à la construction d'un presbytère-chapelle, puis d'une église de pierre.

Certes, les premières années, les paroissiens pouvaient tout juste payer le coût du gros œuvre; mais, plusieurs années plus tard, ils procédaient à l'embellissement de leur église, et les fabriques rivalisaient souvent entre elles pour commander aux sculpteurs, aux peintres et aux orfevrres, des œuvres dans le goût de telle paroisse ou comme à telle autre église. Les contrats d'entreprise sont farcis de ces exemples de mimétisme qui ont marqué l'art religieux du début du XIX ${ }^{\mathrm{e}}$ siècle.

L'exemple est bien connu. On fait d'abord construire les murs, la toiture et les ouvertures; puis les années passent, on commande à un sculpteur un tabernacle, un retable, une chaire, une voûte, un décor sculpté; à un peintre, des tableaux, aux orfèvres, des vases d'argent voire d'or. Ainsi, il s'écoule parfois plus de 20 ans avant que soit achevé l'ornementation complète de l'intérieur d'une église; il va sans dire que plusieurs sculpteurs se succèdent avant que l'ensemble ne soit achevé. C'est ainsi que dans la région de Lanaudière (ou diocèse de Joliette) il n'existe qu'une seule æuvre d'importance sculptée au milieu du XVIII ${ }^{e}$ siècle, soit le tabernacle de Gilles Boivin exécuté en 1759 pour l'église de Berthier. L'on trouve aussi à St-Sulpice un tabemacle sculpté par les frères Francois Noël et Jean-Baptiste-Antoine Levasseur, vers 1750. Cette paroisse, cependant, fait partie $\mathrm{du}$ diocèse de Montréal. Il ne subsiste aucune trace de l'œuvre de ces sculpteurs dans la région. Ils apparaissent donc comme des ouvrages isolés 
à une époque incertaine, marquée par la transition et la pauvreté. Ce n'est qu'à la fin du XVIII' siècle et au début du XIX ${ }^{\mathrm{e}}$ que l'expansion et la fondation des paroisses va provoquer une série de construction d'églises et chapelles qui vont émailler la région de Lanaudière de décors en bois sculpté.

Au début des années 1790, Quévillon commence à exécuter des ouvrages de sculpteur, une fois son apprentissage terminé. Très tôt, il apparaît qu'il fut débordé par les commandes. Au nombre de celles-ci, l'on retrouve une quarantaine d'églises surtout dans la région de Montréal, rive sud, Vallée du Richelieu, l'ouest de l'île mais aussi dans la région de Québec aussi loin que Rivière-Ouelle ou Sainte-Anne-de-la-Pocatière.

Cette expansion du marché à l'époque est telle qu'une seule avenue apparaissait logique pour satisfaire la demande, soit la formation d'apprentis qui à leur tour devenaient des associés du maître, avant de former euxmême des compagnons et ainsi de suite. «Dans l'état actuel de la recherche, écrit John R. Porter, il appert que plus de 80 sculpteurs et apprentis gravitèrent autour du maître de Saint-Vincent-de-Paul et de ses collaborateurs immédiats» ${ }^{\perp}$. On a donc affaire a une sorte de mouvement, ou plutôt d'engouement pour le décor d'église sculpté. L'organisation d'un atelier, sorte d'école de formation ou pendant une période de temps déterminée le maître sculpteur enseigne à ses apprentis les secrets de son art, à charge par ces derniers d'exécuter les travaux que dirige le maître, est l'avenue privilégiée pour satisfaire le demande croissante d'ouvrage sculpté.

\section{Louis-Amable Quévillon (1749-1823) à Ste-Geneviève de Berthier}

C'est à Berthier, la paroisse la plus prospère de la région à la fin du XVIII' siècle, qu'on retrouve Quévillon. Il y travaillera de 1797 à 1810, en plusieurs étapes: en 1797, une cartouche et une chaire; en 1801, une corniche; en 1802, un tombeau d'autel et, en 1810, la chaire et un banc d'œuvre. De ces ouvrages, ne subsiste que le tombeau du maître-autel, les autres ont été remplacés par des ouvrages exécutés des années plus tard par un des meilleurs apprentis du maître des écorres, Amable Gauthier. Nous le verrons plus loin.

\footnotetext{
1 John R. Porter, La Sculpture au Québec, p. 165.
} 


\section{4. À St-Joseph de Lanoraie}

Vers la même époque, en 1797-1799, on lui attribue le tombeau d'autel de St-Sulpice. Puis c'est à St-Joseph de Lanoraie qu'on le retrouve à l'œuvre où, de 1801 à 1805 , il exécute une chaire et un banc d'œuvre pour 1200 livres, un retable et une corniche et divers travaux que la Fabrique mettra plus de 22 ans à payer. En effet, le 15 décembre 1822, un an avant sa mort, Quévillon donne une quittance finale et fait don à la même occasion d'un chandelier pascal. Il aura reçu pour tous ces trâa aux de sculpiure, peinture et dorure, près de 14,000 livres. Ce qui est considérablement pour l'époque. Malheureusement, rien ne subsiste de cet abondant décor et mobilier sculpté; une partie de l'ancien mobilier a probablement été réutilisé dans la nouvelle église construite en 1862-64, mais cette église fut complètement détruite dans un incendie en 1918, puis de nouveau en 1934.

\section{5. À St-Cuthbert}

Par la suite, c'est à St-Cuthbert que Quévillon travaillera. En 1807, i1 entreprend l'exécution de deux tabernacles et autels de chapelles (1 800 livres); en 1808, un retable et une comiche (3 400 livres); en 1809, il travaille à la voûte de l'église (568 livres) et, en 1813-14, on lui paie plus de 6740 livres pour la voûte du sanctuaire, le retable des chapelles et diverses sculptures dans l'église. En 1800, il avait sculpté «six grands chandeliers avec le Christ». Il aura donc exécuté pour plus de 12800 livres d'ouvrage sculpté pour cette église. Fort heureusement, subsistent toujours le Christ et la garniture de six chandeliers, les autels latéraux et divers éléments de sculpture placés autour du cadre de la porte d'entrée de la chapelle du couvent du village (à la fin des années 1880 , ce couvent a été transformé en résidence pour personnes âgées).

\section{6. À St-Antoine de Lavaltrie}

Il est fort probable que Quévillon ait travaillé pour l'église de Lavaltrie. Construite en 1772, elle fut certainement aussi ornementée à la fin du XVIII ${ }^{\mathrm{e}}$ siècle. Mais l'incendie du presbytère en 1910 détruisit tous les livres de comptes sauf le premier qui couvre la période 1767-1785 et où apparaît en 1785 une dépense de 284 livres «pour les chandeliers argentés et autres ouvrages» ${ }^{2}$. Peut-être peut-on attribuer à Quévillon une croix de procession, en noyer, finement sculptée et qui apparaît suffisamment délicate pour être due à son ciseau. Cette croix de procession est toujours conservée

2 En effet, en 1820, on y retrouve le sculpteur Amable Gauthier, ancien apprenti de Quévillon, qui travaillera aussi à St-Cuthbert et à Berthier, sur les traces de Quévillon. Ce qui a été conservé de l'ancienne église est attribué à Amable Gauthier. 
à Lavaltrie. C'est bien peu du décor sculpté à la fin du XVIII' siècle pour l'église qui, après tout, était l'église de la famille seigneuriale, dont le manoir était situé à quelques mètres, au bord du fleuve.

\section{7. À St-Paul de Lavaltrie}

Quévillon a-t-il travaillé pour l'église de St-Paul de Lavaltrie? Nous sommes portés à conclure qu'il aurait exécuté le tabernacle du maître-autel et la garniture de six grands chandeliers et croix qui s'y trouvent toujours, bien que le tabernacle ait subi quelques altérations à son entablement et au baldaquin de la custode. On ne retrouve pas le nom de Quévillon dans le premier livre de comptes (1783-1850) de la fabrique; celui-ci d'ailleurs a été partiellement détruit lors de l'incendie du presbytère en 1903. L'église de St-Paul fut construite en 1803 et terminée en 1804; les paroissiens voulurent sans doute y placer un tabernacle qui soit digne de leur nouvelle église. Or l'analyse stylistique du tabernacle de St-Paul, surtout lorsqu'on le compare au tabernacle des autels latéraux sculptés par Quévillon pour l'église de Boucherville en 1806-1807, présente des similitudes iconographiques frappantes.

D'ailleurs son modèle est sans doute le tabernacle exécuté par Liébert en 1790 pour la chapelle des Sœurs grises, puis pour l'église du Sault-auRécollet et de Vaudreuil en 1792, et de Ste-Rose en 1799. La présence des deux niches de part et d'autre de la custode est un élément architectural tout à fait typique de la production de Liébert. Cependant, le fini de la sculpture du tabernacle de St-Paul est beaucoup plus près des œuvres connues de Quévillon. Selon nous, le modèle serait inspiré des tabernacles de Liébert, mais interprété et exécuté par Quévillon. Quant à la garniture de six grands chandeliers et de la croix, elle aussi puise au répertoire de formes familières à Quévillon. Qu'on les compare par exemple aux chandeliers exécutés par Quévillon pour St-Cuthbert en 1800.

\section{8. À̀ La Visitation de l'île Dupas}

Enfin il est une autre église pour laquelle Quévillon fit des ouvrages de sculpture, soit l'église de La Visitation de l'île Dupas. L'île Dupas est la plus ancienne mission de la région. Dès le milieu du XVII siècle, les missionnaires s'y arrêtaient. L'église actuelle, construite en 1851, est en fait la troisième église. Il est certain que Quévillon travailla à l'ornementation de la deuxième église. L'Annuaire de Ville-Marie, rapporte en effet que Quévillon reçut, en 1781, 1641 livres pour la décoration à l'intérieur, puis 270 
livres en 1800 pour l'argenture d'un Christ et de six grands chandeliers de bois; en 1802, pour un autel à la romaine, 892 livres; en 1820, 2797 livres pour des décorations à l'intérieur de l'église. Des ouvrages exécutés par Quévillon, il ne reste rien; l'église conserve bien un petit autel et un tabernacle, un chandelier pascal et deux bénitiers qui datent probablement du début du XIX ${ }^{\mathrm{e}}$ siècle, mais la facture en est trop gauche pour être du au ciseau de Quévillon. Ils ont dû être exécutés par un apprenti du maître mais ne sont certainement pas de sa main.

Comme on le constate, il subsiste fort peu d'œuvres exécutées par Quévillon lui-même. Aucun ensemble ou décor architectural complet tel que voûte, retable, corniche, chaire, banc d'œuvre, trône curial ou stalles. Quelques pièces de mobilier, dont des tombeaux d'autel à la romaine, tabernacles de maitre-autel et chapelles, garnitures de chandeliers et croix. Tout le reste est disparu dans des démolitions et des incendies successifs. Nous pouvons donc difficilement conclure de façon définitive sur l'importance stylistique de ces œuvres dans la production globale de Quévillon. Qui plus est, il ne subsiste pas de photographies des anciens intérieurs de St-Cuthbert, Lanoraie, St-Sulpice, l'Île Dupas. Il n'y a que deux photos de l'intérieur de l'ancienne église de Lavaltrie où l'on peut deviner des ouvrages de sculpture du genre de ceux exécutés par le maître des Écorres. Ces conclusions cependant ne sont qu'hypothétiques puisque les livres de comptes manquent. Il n'existe pas en outre d'ouvrage exhaustif sur l'œuvre complète de Quévillon. Quand on sait qu'il a sculpté pour plus de 40 églises, on ne peut conclure de façon définitive sur l'importance des œuvres du sculpteur conservées dans la région par rapport à l'œuvre complète de Quévillon.

\section{Joseph Pépin (1770-1842)}

En fait, c'est plutôt du côté d'un autre sculpteur contemporain de Quévillon, qui deviendra en 1815 son associé, que nos recherches connâ̂tront des conclusions plus tangibles. Il s'agit du sculpteur Joseph Pépin. S'il est une région où cet admirable sculpteur s'est illustré, c'est bien la région de Joliette; il a sculpté le décor intérieur complet de l'église St-Jacques de l'Achigan, de St-Ours, du St-Esprit, puis de St-Roch. Mais qui est Joseph Pépin?

Il est né le 19 novembre 1770 et est décédé le 18 août 1842 à l'âge de 72 ans. Marié le 14 février 1803 à Charlotte Stubinger, il fut père de dixneuf enfants dont sept moururent en bas âge. Cinq fils survécurent dont Georges-Henri, Charles, Édouard, René-Clément et Zéphirin. Son beaupère, Stubinger, était venu au Canada avec le régiment Hessois, alors que sa belle-mère était Charlotte de la Broquerie, fille de Joseph Boucher, sieur 
de la Broquerie ${ }^{3}$. Major du premier Bataillon de milice, il fut mêlé aux soulèvements de 1837 de même que son fils Zéphirin, devenu notaire. François Malépart de Beaucourt a peint un portrait de Joseph Pépin, bien que ce portrait demeure aujourd'hui introuvable.

Joseph Pépin fut aussi l'apprenti de Philippe Liébert et très tôt il travailla avec Quévillon. En fait les deux hommes se connaissaient depuis 1791, année où Pépin résidait à St-Vincent de Paul. En 1803, il sculptait à l'église de Boucherville avec Quévillon; en 1805 et 1810, on les retrouve à l'église de Beloeil et à l'église des Cèdres. En 1815, Quévillon, Pépin, René St-James dit Beauvais (1785-1837) et Paul Rollin (1789-1855) signent un contrat d'association. Bien que celle-ci ne dure formellement que jusqu'en 1817 , ils continuèrent néanmoins de travailler ensemble, comme à Verchères en 1817.

\section{0. À St-Antoine de Lavaltrie}

Après la mort de Quévillon en 1823, Pépin lui survécu jusqu'en 1842, continuant de sculpter avec ses apprentis, ses fils et son neveu, François Pépin. On peut facilement dénombrer plus d'une douzaine d'églises où il a travaillé, soit seul ou avec ses fils et neveu, soit avec ses associés ou collègues Quévillon, Beauvais ou Rollin. Où apparaît-il d'abord dans la région de Lanaudière? À nul autre endroit qu'à St-Antoine de Lavaltrie. En fait, c'est là qu'il s'y marie le 14 février 1803. Comme il a été dit, les anciens livres de comptes de Lavaltrie furent détruits et l'ancienne église de 1772, démolie en 1869 et son mobilier dispersé (une partie vendue à l'église de St-Théodore de Chertsey, autrefois de Montcalm). Comme il arrivait fréquemment que les sculpteurs s'installaient dans la paroisse où ils travaillaient, puis se transportaient dans une autre paroisse au fil des commandes, on peut donc présumer qu'en 1803 Joseph Pépin travaillait à l'église de Lavaltrie.

\section{1. À St-Jacques de l'Achigan}

En 1816, c'est à St-Jacques de l'Achigan que Joseph Pépin entreprend des travaux d'ornementation majeurs pour la somme rondelette de 28548 livres. Il s'engage par contrat notarié à y exécuter: «une voûte, les trois retables, les deux autels, le banc d'œuvre, la chaire, les balustres, le chandelier pascal, les corniches, une garniture de chandelier pour chaque autel, trois croix et deux autres chandeliers semblables pour le banc d'œuvre, les

3 Raymonde Gauthier, «Joseph Pépin», Dictionnaire biographique du Canada, t. VII, Québec, Presses de l'Université Laval, 1988, p. 742-743.. 
boisures du chœur, les crédences, les tabernacles pour les chapelles, trois cadres sculptés pour les trois grands tableaux, deux pupitres pour les grands livres de chant, deux trônes et les boisures du chœur, neuf tabourets, de même que la peinture et la dorure» de l'ensemble de ce décor et mobilier sculptés. Le contrat prévoit la nourriture de quatre hommes pendant un an et demi. En 1820, il agrandit le jubé et, en 1822, François Pépin, «compagnon sculpteur», reçoit 391 livres pour ce travail. En 1824, puis en 1831, un second contrat pour la construction d'un autre jubé lui rapporte 4000 livres. Il cxécuite en oütie six chlandeliers et une croix argentée pour la somme de 240 livres.

Malheureusement un incendie détruisit, en 1914, la première église de St-Jacques. Cependant on réussit à sauver une partie du mobilier, soit des chandeliers, les deux crédences, les deux autels latéraux et le chandelier pascal. Le musée de Joliette conserve l'un des tabernacles et deux statuettes d'une naïveté charmante. Dans la paroisse voisine de St-Roch, Pépin avait entrepris en 1815 des travaux d'égale importance: «un chandelier pascal, une voûte avec fausse coupole et corniche, un retable, les cadres des trois tableaux, les crédences ou consoles du sanctuaire, des reliquaires, une chaire, deux trônes (qui servirent de modèles pour celui de St-Jacques), un banc d'œuvre avec un dais, trois tabernacles et trois autels». Malheureusement un incendie détruisit en janvier 1917 ce merveilleux ensemble qui nous est connu par un relevé photographique complet, réalisé quelques mois avant l'incendie. Seuls subsistent un reliquaire et probablement le chandelier pascal, conservés dans des collections privées à Montréal.

\section{2. À St-Ours du St-Esprit}

Il en alla de même pour l'église de St-Ours du St-Esprit construite en 1810. En 1820, Joseph Pépin y travaille à la sculpture de la voûte, puis, de 1825 à 1835 , il exécute un chandelier pascal et des garnitures de chandeliers semblables à ceux de St-Jacques. L'ancienne église de St-Esprit fut démolie et remplacée par une seconde église en 1902, laquelle fut détruite par le feu en 1931. Il ne subsiste presque rien de l'ancien décor sculpté. Le Musée de Joliette conserve un ancien lustre qui pourrait provenir de cette église; le Musée d'art de St-Laurent, une porte à panneaux sculptés provenant du sanctuaire; tout le reste est disparu.

Voilà donc un sculpteur dont les œuvres exécutées à St-Roch et à StJacques donnaient une juste mesure du talent et du charme de son habileté. Encore là, il ne subsiste que quelques pièces de mobilier de l'œuvre sculpté de Pépin dans la région. Mais si l'œuvre de Pépin a quasi complètement 
disparu, celui du sculpteur Amable Gauthier, apprenti de Quévillon, est fort heureusement plus accessible. Qui est Amable Gauthier?

\section{Amable Gauthier (1792-1876) à St-Antoine de Lavaltrie}

Né en 1792 à St-Jean-Baptiste de Nicolet, il décéda en 1876 à l'âge de 84 ans et fut inhumé à St-Barthélemi. Jeune orphelin, il aurait été placé par son oncle à l'atelier de Quévillon ${ }^{4}$. Capitaine de milice en 1833, il cacha deux proscrits dans sa maison lors des soulèvements de 1837. Il fut père d'au moins quinze enfants. L'un de ses fils, Louis-Zéphirin, deviendra architecte prolifique, faisant les plans de plus de 100 églises. Fort talentueux, en fait le meilleur élève de Quévillon, il vole très tôt de ses propres ailes. Ainsi, en 1820, il signe au Manoir Seigneurial de Lavaltrie, devant le notaire Barthélemy Joliette, un contrat avec la fabrique par lequel il s'engage à refaire une nouvelle voûte, le retable du maître-autel avec les boisures, corniches et autres ornements du chœur, deux petits retables pour les chapelles, une corniche pour la nef, un banc d'œuvre, le chapeau de la chaire, l'escalier et la rampe, enfin un jubé, le tout peint blanc et «doré avec de l'or", pour la somme de 14000 livres. Le contrat décrit le Sieur Gauthier comme architecte et sculpteur demeurant à Yamachiche. Il reste peu de chose de Gauthier à Lavaltrie: une garniture de chandelier et une croix, ainsi que la banquette du banc d'œuvre, adossée derrière le maîtreautel, du côté de la sacristie; une rosace de bois au plafond de la sacristie provient sans doute de la voûte de l'ancienne église.

\section{4. À Ste-Geneviève de Berthier}

Dès l'année suivante, Gauthier est à Berthier. À cet endroit il s'engage avec Alexis Milette, le 24 novembre 1821, «à faire la voûte, la corniche selon l'ordre corinthien, le tout peint et doré avec les ornements selon le plan présenté par eux-mêmes»" ${ }^{5}$. Un an plus tard l'ouvrage est terminé et le 17 août 1823 les marguilliers retiennent à nouveau leurs services pour «le sanctuaire, le retable, la boiserie, l'autel, les marches, les stalles, deux jubés, une corniche, le banc d'œuvre et un baptistaire dans le goût de celui de Boucherville». Ils les chargent également de «reporter le vieux retable à une chapelle, d'en faire un autre pour l'autre chapelle, de rétablir la chaire dans le même goût que celle de l'église Ste-Marie». Trois ans plus tard, le 11 mai 1826 , nouveau contrat pour terminer la décoration de l'église; il

4 Jean Coutu, «Amable Gauthier», Dictionnaire biographique du Canada, t. X, Québec, Presses de l'Université Laval, 1972, p. 329.

5 Alexis Milette (1793-1870) était également de Yamachiche. Dans son atelier, il formait aussi des apprentis. 
s'agit «des petits tabernacles et autels des chapelles, des confessionnaux, d'argenter les vieux chandeliers d'argent, de faire un chandelier pascal et autres articles». L'ensemble du décor est terminé vers 1830. Il constitue l'œuvre principale d'Amable Gauthier et d'Alexis Milette qui nous soit parvenue sans transformations majeures ${ }^{6}$. Gauthier reviendra de nouveau à Berthier, cette fois en 1844, pour travailler à l'éloignement des murs latéraux de l'église, à la construction des tribunes latérales et à la réparation du portique et du clocher. L'église de Berthier est donc unique en ce qu'elle contient le seul décor sculnté d'originge qui soit complet dans la région.

\section{5. À St-Cuthbert}

Puis en 1827, il est à St-Cuthbert, là même où ont travaillé Philippe Liébert en 1791-98, et Quévillon, son maître, en 1807-1808. Il y a une filiation remarquable dans cette église. Il y exécute un triple portique d'ordre ionique puis, en 1830, la statue monumentale du saint patron (qu'on peut encore apercevoir devant l'église). En 1835, il ajoute des corniches dans la nef et des omements à la voûte et exécute un remarquable entablement dont le fronton repose sur deux colonnes torses pour servir de retable à l'autel de Liébert. En 1840-41, il reçoit encore des paiements pour des ouvrages "pour le chœur». Certains des ouvrages de Gauthier ont été réinstallés dans l'église actuelle de St-Cuthbert, dont des colonnes torses du retable et de la corniche et frise du sanctuaire. On peut facilement les distinguer du reste de la nouvelle église tant la sculpture est grasse, quasi «boudinée».

\section{6. À Ste-Élisabeth}

En 1835, il est à Ste-Élisabeth où il exécute un superbe maître-autel, qui reste l'un des plus beaux de toute son œuvre sculptée; il y exécute en particulier trois statuettes de saints tout à fait typique de son style de sculpture, grasse, abondante, quasi «italienne»; il sculpte également un chandelier pascal dans le goût classique des œuvres de l'atelier de Quévillon. Cependant tout le reste de son travail pour l'église de Ste-Élisabeth disparaît dans la démolition de l'église en 1902, puis lors de la démolition de la seconde église en 1949, fermée depuis $1931^{7}$.

6 Les églises et le trésor de Berthierville. Coll. «Les Retrouvailles», no 1, p. 8-9.

7 Un panneau de la frise est conservé au Musće de Joliette. 


\section{7. À St-Joseph de Lanoraie}

En 1837, Gauthier reçoit 72 livres de la fabrique de Lanoraie pour «plan et voyage», sans doute pour une réparation exécutée à l'église. En 1843-44, il exécute pour l'église de St-Sulpice une garniture de chandeliers semblable à celle de Lavaltrie (elle s'y trouve encore).

\section{8. À St-Barthélemi}

Toujours en 1843, il est marguiller à St-Barthélemi. En 1845, il passe un contrat avec la fabrique pour construire une nouvelle église; il est alors décrit comme entrepreneur. Malheureusement cette nouvelle église sera incendiée le 14 novembre 1865. En 1850, il exécute une garniture de six chandeliers et une croix pour cette église; en 1855, une jolie statuette de la Madone et de l'Enfant. Enfin, en 1870, la fabrique lui paiera $300 \$$ pour une chaire à prêcher que Gauthier avait déjà exécutée. Elle existe toujours, conservée dans l'église, de même que la garniture et la statuette de la Madone et de l'Enfant. Certains ouvrages mentionnent qu'il aurait aussi travaillé à St-Paul. Nous en doutons; les livres de comptes ayant été détruits, rien dans l'église actuelle n'apparaît être dû au ciseau de Gauțhier.

Toutefois l'église de St-Edmond conserve une croix de procession dont le style de sculpture apparaît être tout à fait une œuvre de Gauthier, tant elle est semblable à la croix de la gamiture de Berthier ou de St-Barthélemi. Elle provient certainement d'une ancienne paroisse qui lui en fit don, comme il était courant à l'époque.

En effet, plusieurs nouvelles paroisses du Nord du diocèse recevaient en don du mobilier désuet des plus anciennes paroisses de la région (c'està-dire des paroisses du Sud) lorsque ces églises étaient démolies ou incendiées pour faire place à de nouvelles constructions.

Ainsi on voit sur une ancienne photo de l'intérieur de l'église de SteMélanie une chaire qui semble tout droit sortie de l'atelier de Joseph Pépin. Bien sûr elle ne s'y trouve plus aujourd'hui; mais elle a dû provenir d'une plus ancienne paroisse. De même à l'église de St-Calixte, on conserve deux autels latéraux et un tombeau de maître-autel, qui eux aussi

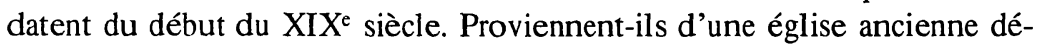
molie comme St-Henri de Mascouche par exemple ou Ste-Anne des Plaines, où ont-ils été sauvés d'un incendie comme celui de Lanoraie ou St-Esprit, et donnés à cette petite paroisse de colonisation?

On ne peut pas l'affirmer avec certitude; toutefois leur style et leurs motifs iconographiques les placent directement dans la lignée des œuvres sorties de l'atelier de Quévillon et de ses associés. Il en va de même pour cet autel galbé qui apparaît sur une photo de l'intérieur de la chapelle du 
couvent de St-Félix de Valois, construite en 1875 et aujourd'hui démolie. Il date certainement du début du siècle.

\section{Des apprentis, des disciples}

Nous avons dit un mot de Liébert, Quévillon, Pépin et Gauthier. Ce sont des maitres qui ont formé chacun des apprentis. Nous sommes attardés sur leur œuvre dans le diocèse parce qu'ils sont les meilleurs sculpteurs à avoir travaillé dans la région. Il en est toutefois d'autres qui se sont situés dans leur sillage et dont on retrouve la trace de façon moins élaborée. Parfois, il faut le dire aussi, le talent se faisait plus rare. Retenons le nom de Vincent Chartrand (mort en 1863) et de Pierre Salomon Benoît dit Marquette (né vers 1800): deux apprentis de Quévillon en 1819 qui exécuteront en 1831 pour l'église de l'n̂le Dupas les stalles du chœur et deux confessionnaux pour 624 livres. Ce sont les seules œuvres qu'on leur connaisse dans la région. Ces confessionnaux sont toujours à l'île Dupas.

Il y eut aussi Pierre Guibord (né en 1799), apprenti de Quévillon en 1816, qui exécuta des autels latéraux pour l'églisc dc Ste-Élisabeth (toujours conservés), et qui fut engagé en 1826 à St-Paul de Lavaltrie pour faire «une corniche et peinturer la voûte» et à nouveau en 1827 pour «faire la boisure du chœur, deux trônes, un banc d'œuvre (conservé au Musée de Joliette après avoir servi d'autel à Ste-Julienne), deux retables dans les chapelles, un baldaquin semblable à celui de l'église du grand St-Esprit, un chandelier pascal, un jubé, deux petits autels semblables à ceux de l'église de Ste-Élisabeth, le tout pour 15,000 livres».

Ajoutons que tous ces ouvrages auraient dû être exécutés par Chrysostome Perreault, originaire de St-Paul, qui s'y était obligé par contrat en 1821. Perreault avait été l'apprenti d'Amable Charron en 1810, et Charron l'apprenti de Quévillon quelques dix ans plus tôt. Perreault avait dû se désister cinq ans plus tard, en 1826, n'ayant pu compléter les ouvrages à temps. Seule la voûte semble avoir été terminée puisque Guibord s'engage à la peindre en 1826. La sculpture de Guibord est mince, le volume de ses meubles un peu lourdaud. Parmi les autres apprentis, mentionnons Pierre Viaud, qui exécuta pour la première église de St-Lin un maître-autel et une garniture conservés au Musée de Joliette. Peu d'autres œuvres ont été identifiées ou attribuées à ce sculpteur dont le ciseau est un peu frustre et sans grande poésie. Ajoutons le nom de Paul Lefebvre, auteur à Ste-Élisabeth d'une garniture de chandeliers, conservée à l'évêché de Joliette. Une sculpture plus grèle mais douce et patinée.

Voilà donc un survol rapide de l'œuvre de l'atelier de Quévillon dans la région de Lanaudière. Comme on le constate, il couvre toute la première moitié du XIX ${ }^{\mathrm{e}}$ siècle. Ce qui étonne dans l'abondance de cette production, 
c'est qu'il subsiste fort peu de chose. quelques autels, garniture de chandeliers, quelques éléments ou ornements sauvés de la démolition ou des incendies, et souvent dispersés. Quelque-uns encore exposés au risque d'une destruction comme ceux de l'île Dupas, dans un arrière de sacristie en bois, ou ceux de Notre-Dame-de-la-Merci.

Il faut le préciser, l'étude de la sculpture décorative est demeurée le parent pauvre des historiens d'art; pourtant, pendant plus de 100 ans, ce fut la forme la plus originale d'expression artistique au Québec, à une époque où les Canadiens français s'enorgueillaient à juste titre du décor doré de leur église. 